\title{
Bioinformatics analysis of proteomics profiles in senescent human primary proximal tubule epithelial cells
}

\author{
Yang $\mathrm{Lu}^{1 \dagger}$, Jingchao Wang ${ }^{1 \dagger}$, Chen Dapeng ${ }^{1,2}$, Di Wu${ }^{1}$, Guangyan Cai ${ }^{1}$ and Xiangmei Chen ${ }^{{ }^{*}}$
}

\begin{abstract}
Background: Dysfunction of renal tubule epithelial cells is associated with renal tubulointerstitial fibrosis. Exploration of the proteomic profiles of senesced tubule epithelial cells is essential to elucidate the mechanism of tubulointerstitium development.

Methods: Primary human proximal tubule epithelial cells from passage 3 (P3) and passage 6 (P6) were selected for evaluation. EdU and SA- $\beta$-galactosidase staining were used to detect cell senescence. p53, p21, and p16 were detected by Western blot analysis. Liquid chromatography mass spectrometry (LC-MS) was used to examine differentially expressed proteins (DEPs) between P6 and P3 cells. The expression of DEPs was examined by Western blot analysis. Bioinformatics analysis was performed by protein-protein interaction and gene ontology analyses.

Results: The majority of tubule cells from passage 6 (P6) stained positive for SA- $\beta$-galactosidase, whereas passage 3 (P3) cells were negative. Senescence biomarkers, including p53, p21, and p16, were upregulated in P6 cells relative to P3 cells. EdU staining results showed a lower rate of EdU positive cells in P6 cells than in P3 cells. LC-MS was used to examine DEPs between P6 and P3 cells. These DEPs are involved in glycolysis, response to stress, cytoskeleton regulation, oxidative reduction, ATP binding, and oxidative stress. Using Western blot analysis, we validated the downregulation of AKR1B1, EEF2, EEF1A1, and HSP90 and the up-regulation of VIM in P6 cells seen in the LC-MS data. More importantly, we built the molecular network based on biological functions and protein-protein interactions and found that the DEPs are involved in translation elongation, stress, and glycolysis, and that they are all associated with cytoskeleton regulation, which regulates senescent cell activities such as apoptosis and EMT in tubule epithelial cells.

Conclusions: We explored proteomic profile changes in cell culture-induced senescent cells and built senescenceassociated molecular networks, which will help to elucidate the mechanisms of senescence in human proximal tubule epithelial cells.
\end{abstract}

Keywords: Proximal tubule cells, Senescence, Proteomics, Network

\section{Background}

Aging and aging-related diseases are associated with various health problems worldwide. Renal aging begins around approximately 40 years old and is accompanied by decreased renal blood flow ( $10 \%$ per year). The decline in renal function and susceptibility to age-related

\footnotetext{
*Correspondence: xmchen301@126.com

${ }^{\dagger}$ Equal contributors

'Department of Nephrology, Chinese PLA General Hospital, Chinese PLA Institute of Nephrology, Beijing Key Laboratory of Kidney Disease, State Key Laboratory of Kidney Diseases, National Clinical Research Center for Kidney Diseases, General Hospital of PLA, Fuxing Road 28, Beijing 100853, P.R. China Full list of author information is available at the end of the article
}

renal insufficiency may contribute to chronic progressive kidney failure. Senescence of tubule epithelial cells involves multiple complex activities including apoptosis, cell shape enlargement, decreased motility, aberrant energy metabolism, and epithelial-to-mesenchymal transition (EMT), and it is the major cause of renal tubulointerstitial fibrosis [1] and kidney failure. Exploring the mechanism of senescent tubule epithelial cell activities will aid us in understanding the pathogenesis of renal PTEC senescence.

The known senescence-associated cell activities are mainly based on changes in protein components. It has been reported that the upregulation of nuclear factor 
kappa B (NF-kB), tumor growth factor beta (TGF- $\beta$ ) and hypoxia-inducible factor (HIF) affect tubule epithelial cell proliferation, cellular apoptosis and EMT, which is associated with senescence in tubule epithelial cells $[2,3]$. However, there is still a lack of large-scale proteomic analyses that have explored aging-related proteins and mechanisms. Therefore, we performed label-free quantitative proteomics and explored proteomic profiles in senescent human proximal tubule epithelial cells (PTECs) to identify the molecular mechanism underlying senescenceassociated cell activities in PTECs.

\section{Methods}

\section{Isolation and culture of human primary tubular cells}

Segments of macroscopically and histologically normal renal cortex were obtained under aseptic conditions from patients undergoing nephrectomy for small $(<6 \mathrm{~cm})$ tumors in the Department of Urology, Chinese PLA General Hospital. Patients were accepted into the study if they had no history of renal or systemic disease associated with tubulointerstitial pathology. Tubular fragments were derived from the segments of renal cortex by collagenase digestion and were isolated by centrifugation in $45 \%$ Percoll (Pharmacia, Uppsala, Sweden). The PTECs were re-suspended in a 1:1 ( $\mathrm{vol} / \mathrm{vol})$ mixture of Dulbecco's modified Eagle's $\left(\mathrm{GIBCO}^{\mathrm{TM}}\right.$ Invitrogen, Barcelona, Spain) and Ham's F-12 media (Hyclone, USA) supplemented with $10 \%$ heat-inactivated fetal bovine serum (FBS) $\left(\right.$ GIBCO $^{\mathrm{TM}}$ Invitrogen, Barcelona, Spain), 10 ng/ml EGF (Peprotech, Rocky Hill, USA), $5 \mathrm{mg} / \mathrm{ml}$ human transferrin, $5 \mathrm{mg} / \mathrm{ml}$ bovine insulin (all from Sigma, St. Louis, MO, USA), $100 \mathrm{U} / \mathrm{ml}$ penicillin, and $100 \mu \mathrm{g} / \mathrm{ml}$ streptomycin (Invitrogen, New York, NY, USA). Passage 3 was defined as the young control, and passage 6 was defined as cellular senescence.

\section{Immunofluorescence}

The PTEC biomarker cytokeratin 18 was detected by immunofluorescence. Cells were fixed in $4 \%$ paraformaldehyde and permeabilized with $1 \%$ Triton X-100 buffer. Cells were then incubated with anti-CK18 antibody (Zhongshan Golden Bridge Bio-technology, Beijing, China) and DAPI (Sigma-Aldrich, St. Louis, MO, USA) for nuclear staining. Cells were examined using a Nikon fluorescence microscope (Japan).

\section{SA- $\beta$-gal staining}

Cells were fixed with $2 \%$ formaldehyde and $0.2 \%$ glutaraldehyde for $15 \mathrm{~min}$ and stained with freshly prepared senescence-associated $\beta$-galactosidase (SA- $\beta$-gal) $(1 \mathrm{mg} /$ $\mathrm{mL} \mathrm{X}$-gal, $40 \mathrm{mM}$ citric acid/sodium phosphate ( $\mathrm{pH}$ 6.0), $5 \mathrm{mM}$ potassium ferrocyanide, $5 \mathrm{mM}$ potassium ferricyanide, $150 \mathrm{mM} \mathrm{NaCl}$, and $2 \mathrm{mM} \mathrm{MgCl}_{2}$ ) overnight at $37{ }^{\circ} \mathrm{C}$ without $\mathrm{CO}_{2}$. The cells were then examined under a microscope.

\section{5-ethynyl-2'-deoxyuridine (EdU) proliferation assay}

Proliferative activity was detected using an EdU labeling kit (Roche Ltd, USA) following the manufacturer's recommendations. Fluorescent images were obtained by florescence microscopy.

\section{Western blot analysis}

Antibodies against p53 (Abcam, Cambridge, UK), p53, AKR1B1, EEF1A1, EEF2, HSP90 (Proteintech Group Inc.), p21, and p16 (Cell Signaling Technology, Danvers MA, USA) were used for Western blot analysis. $\beta$-Actin (Sigma-Aldrich, St. Louis, MO, USA) served as a control. Approximately $30 \mu \mathrm{g}$ of protein were subjected to $12 \%$ sodium dodecyl sulfate-polyacrylamide gel electrophoresis (SDS-PAGE). After incubation in primary and secondary antibodies, images were acquired using an Opti-Chemi 600 (UVP Inc., Upland, CA, USA).

\section{Label-free quantitative proteomics}

Protein $(50 \mu \mathrm{g})$ was separated by $12 \%$ SDS-PAGE. Gels were stained with R250 Coomassie Brilliant Blue. Each lane of the gel was cut into four fragments, and each fragment was trypsin-digested as described previously [4]. Peptides were analyzed using twodimensional (2-D) liquid chromatography mass spectrometry (LC-MS) (XEVO QTOF, Waters Corp., Manchester, UK). Samples were separated on a 180$\mu \mathrm{m} \times 50-\mathrm{mm}$ Symmetry C18 $5 \mu \mathrm{m}$ (Waters Corp., Manchester, UK) reversed-phase trap column in the first dimension with Solvent A (200 mM ammonium formate, $\mathrm{pH} 10.0)$ and solvent $\mathrm{B}(\mathrm{CH} 3 \mathrm{CN})$. Five different solvent plugs set automatically by Masslynx 4.1 were applied to elute the fractions sequentially. In the second dimension, peptides were eluted with a nanoACQUITY system equipped with a C18 column (75 $\mu \mathrm{m} \times 100 \mathrm{~mm}$; Waters Corp.) with solvent A (water) and solvent $\mathrm{B}(\mathrm{CH} 3 \mathrm{CN})$. The procedure and data analysis were similar to those in our previous study [5]. The column temperature was maintained at $35{ }^{\circ} \mathrm{C}$. Two hundred femtomolesl $/ \mu \mathrm{L}$ of [Glu1] fibrinopeptide B was applied as the lock mass with a constant flow rate of $300 \mathrm{nl} / \mathrm{min}$. Each sample was detected in triplicate. The spectral acquisition time in each mode was $0.6 \mathrm{~s}$. In the low energy MS mode, data were collected at a constant collision energy of $6 \mathrm{eV}$. In the elevated energy MS mode, the collision energy was increased from 15 to $36 \mathrm{eV}$. Each sample group contained three replicates that were combined for expression profile analysis by PLGS 2.4. The precursor and fragment ion tolerance were determined automatically. The default protein identification 
criteria included a maximal protein mass of $500,000 \mathrm{Da}$ and a detection of at least three fragment ions per peptide, seven fragment ions per protein, and one peptide per protein. Fixed modification of carbamidomethyl- $\mathrm{C}$ and the detected variable modifications, including acetylation (N-terminus), deamidation (N/Q) and oxidation of methionines, were selected. At most, two missed cleavages and a false positive rate of $4 \%$ were allowed. Normalization was performed using the auto-normalization function, [6] which exhibited an effect similar to the internal standard [7]. The NCBI human database (released in March, 2012) was used as a reference database. Only those proteins identified in at least two of three injections and demonstrating fold changes $>1.5$ were considered differentially expressed proteins (DEPs).

\section{Bioinformatics analysis}

Data analysis was performed using MAS 3.0 (http://bioinfo.capitalbio.com/mas3/), BiNGO and 2.44 STRING 9.0 (http://string.embl-heidelberg.de) software. The ClueGo and BiNGO 2.44 software and plug-ins for Cytoscape 2.7 were used to analyze the biological functions. String and the MAS 3.0 system were used for protein-protein interaction (PPI) analysis (score $>0.6$ ) and PPI network building. Cytoscape 2.7 was used to modify the network.

\section{Ethics statement}

The study protocol was approved by the Ethics Committee of PLA general hospital of China. Written informed consent was obtained from all study participants. The diagnosis of renal cancer was made based on results of renal imaging testing and pathological examination.

\section{Results}

Tubule cells from passage 6 exhibit an obvious senescence phenotype

We first identified primary proximal tubule cells by confirming the expression of cytokeratin 18 (CK18). Immunofluorescence of CK18 in the cytoplasm confirmed the purity of tubule cells (Fig. 1a). Next, SA- $\beta$-gal staining was performed to detect senescence. Nearly all tubule cells from the passage 6 (P6) group stained positive for SA- $\beta$-gal (Fig. 1b). Moreover, the expression of senescence biomarkers including p53, p21, and p16 were detected and upregulated in the P6 group compared to passage 3 (P3) cells (Fig. 1c). EdU staining revealed that a lower rate of positivity in P6 cells compared with P3 cells (Fig. 1d). Therefore, P6 tubule cells were defined as senescent.
LC-MS results showed that DEPs in the senescent PTECs were associated mainly with metabolism, cytoskeleton regulation, oxidative reduction, and stress

LC-MS was used to examine the DEPs between P3 and P6 renal tubule epithelial cells. Thirty-four proteins were downregulated and 36 proteins were upregulated in P6 cells compared to P3 cells (Tables 1 and 2). We then applied two tools to analyze the functions of these DEPs. CLUEGO analysis showed that these proteins are involved in the regulation of cellular amino acid metabolic processes, apoptosis, actin-mediated cell contraction, and glucose catabolism (Fig. 2a). BinGO analysis revealed additional biological functions, including glycolysis, response to stress, cytoskeleton regulation, oxidative reduction, adenosine triphosphate (ATP) binding, and oxidative stress (Fig. 2b). These DEPs regulate biological functions related to the process of senescence in PTECs. Moreover, we also validated the expression of DEPs by Western blot analysis (Fig. 2c). We confirmed that AKR1B1, EEF2, EEF1A1, and HSP90 were downregulated and that VIM was upregulated in P6 cells, which is consistent with our proteomic results.

\section{Biological functions, including translation elongation, stress, and glycolysis, were all associated with cytoskeleton regulation based on PPI in senescence- associated molecular networks}

To better explore the mechanisms involved in PTEC senescence, we built molecular networks based on PPI (Fig. 3). In the network, EEF1A1 and EEF2 regulate eukaryotic translation elongation, and GAPDH, ALDOA, ENO2, LDHA, and PKLR are involved in glycolysis. Other DEPs, such as ACTN2, VIM, ANXA2, MSN, and GSM mediate cytoskeleton regulation. HSP90B1, HSP90AA1 and HYOU1 are associated with oxidative stress. More importantly, translation elongation, stress and glycolysis were all related to cytoskeleton regulation, which was associated with regulation of PTEC apoptosis and EMT.

\section{Discussion}

In this study, the specific protein changes involved in human PTEC senescence were explored. The altered proteins were found to be involved in regulating senescence-associated biological functions including cytoskeleton regulation, glycolysis, stress and metabolism. More importantly, these biological functions can affect each other via PPI, which provide new insights on the mechanism of senescence in PTECs.

Aberrant energy metabolism, such as glucose hysteresis, is an important cause of aging. In this study, we determined that key enzymes such as PKLR, ALDOA, GAPDH, and LDHA were disrupted and contributed to a disturbance in glycolysis. ALDOA is a key enzyme that 
A

CK18

DAPI

Merge
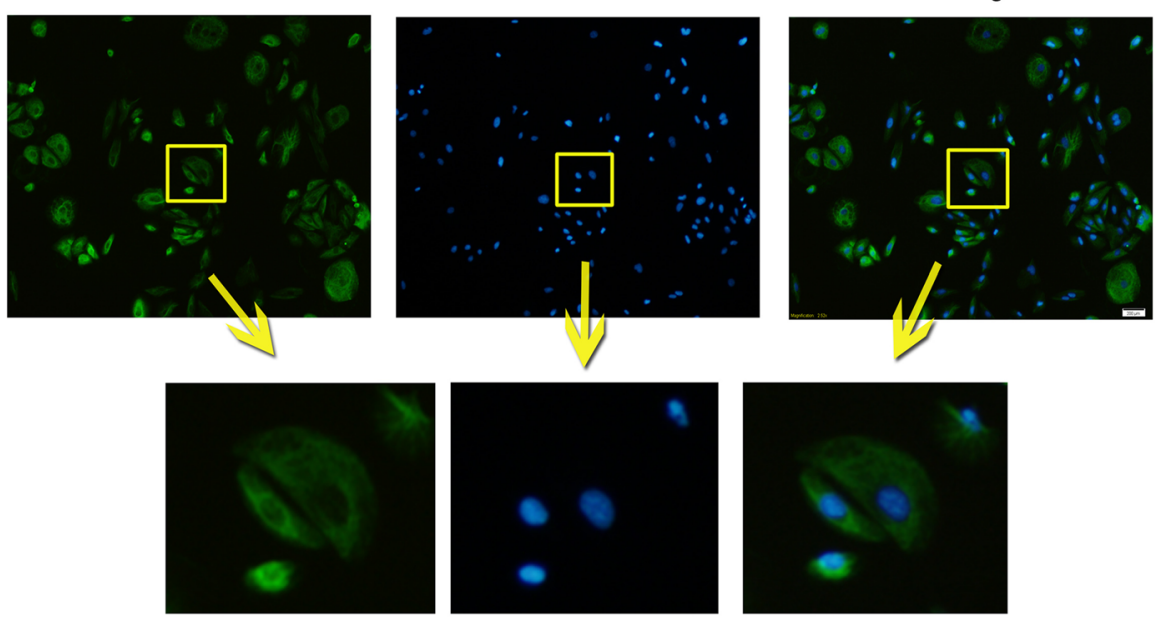

B

P3

C

P6
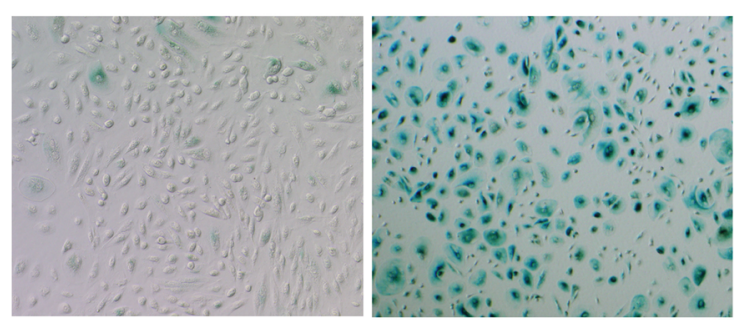

C

P3 P6

p21

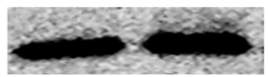

$\mathrm{p} 53$

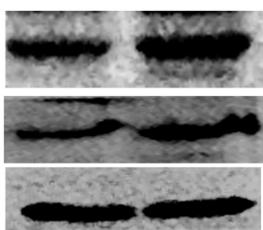

D

P3

P6
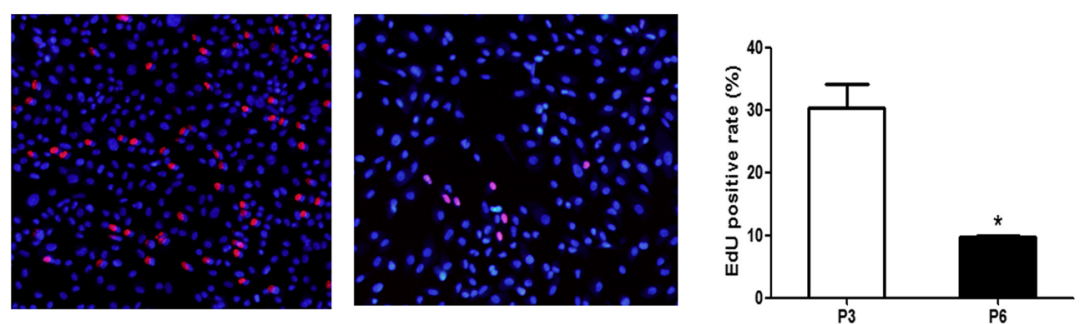

Fig. 1 Tubule cells from passage 6 exhibit an obvious senescence phenotype. a Primary PTEC was identified by immunofluorescence of CK18. b $\beta$-galactosidase staining was performed to detect senescence. Nearly all tubule cells from the passage 6 (P6) group stained positive for $\beta$ galactosidase. c Senescence biomarkers including p53, p21, and p16 were detected by Western blot and upregulated in the P6 group compared to P3 cells. $\mathbf{d}$ EdU staining results showed that there was lower positive rate in the P6 group than P3 group

catalyzes the reversible conversion of fructose-1,6bisphosphate to glyceraldehydes-3-phosphate (GAPDH) and dihydroxyacetone phosphate in glycolysis [8]. LDHA catalyzes the interconversion of pyruvate and lactate PKLR, and, as a pyruvate kinase, catalyzes the transphosphorylation of phosphoenolpyruvate into pyruvate and ATP, which is the final step of glycolysis [9]. In P6 cells, low-expression of LDHA, GAPDH and PKLR may be involved in reduced glycolytic function, which could subsequently promote glucose hysteresis. Renal tubule epithelial cells are high-energy-demanding polarized epithelial cells [10]. In diabetic patients, senescent tubule epithelial cells may be prone to glucose metabolic dysfunction under hyper-glucose conditions.

Stress plays a crucial role in senescence. In our study, we revealed that most stress-associated proteins including HYOU1, HSP90, NQO1, and XRCC were downregulated in aging renal tubule epithelial cells. Specifically, HYOU1 is associated with endoplasmic reticulum stress [11], HSP90 is a stress-induced protein that participates in stress resistance [12], NQO1 protects against oxidative stress induced by a variety of metabolic situations, and XRCC5 (Ku80) is crucial for stress-induced DNA double-strand break repair [13]. These proteins may play 
Table 1 The downregulated DEPs in P6 tubule cells detected by LC-MS (fold change >1.5)

\begin{tabular}{|c|c|c|c|c|}
\hline Accession & Description & Protein & P6/P3 ratio & $\begin{array}{l}\text { Standard } \\
\text { deviation }\end{array}$ \\
\hline NP_001074007.2 & aldo keto reductase family 1 member B15 & AKR1B15 & 0.01 & 0.006 \\
\hline NP_001002858.1 & annexin A2 & ANXA2 & 0.12 & 0.025 \\
\hline NP_001619.1 & aldose reductase & AKR1B1 & 0.17 & 0.026 \\
\hline XP_933678.1 & PREDICTED POTE ankyrin domain family member I & POTEI & 0.18 & 0.15 \\
\hline NP_002037.2 & glyceraldehyde 3 phosphate dehydrogenase & GAPDH & 0.30 & 0.03 \\
\hline NP_000289.1 & pyruvate kinase isozymes $R \mathrm{~L}$ & PKLR & 0.37 & 0.016 \\
\hline NP_003290.1 & endoplasmin precursor & HSP90B1 & 0.41 & 0.006 \\
\hline NP_002256.2 & importin subunit beta 1 & KPNB1 & 0.41 & 0.02 \\
\hline NP_003325.2 & ubiquitin like modifier activating enzyme 1 & UBA1 & 0.43 & 0.007 \\
\hline NP_005339.3 & heat shock protein HSP 90 alpha & HSP90AA2 & 0.44 & 0.004 \\
\hline NP_001153706.1 & sodium potassium transporting ATPase subunit alpha 1 & ATP1A1 & 0.48 & 0.019 \\
\hline NP_001171651.1 & glucose 6 phosphate isomerase & GPI & 0.61 & 0.009 \\
\hline NP_001393.1 & elongation factor 1 alpha 1 & EEF1A1 & 0.66 & 0.009 \\
\hline NP_945189.1 & protein glutamine gamma glutamyltransferase 2 & TGM2 & 0.63 & 0.017 \\
\hline NP_065843.3 & neutral cholesterol ester hydrolase 1 & AADACL1 & P3 & \\
\hline NP_000687.3 & 4 trimethylaminobutyraldehyde dehydrogenase & ALDH9A1 & P3 & \\
\hline NP_004299.1 & rho GTPase activating protein 1 & ARHGAP1 & P3 & \\
\hline NP_001719.2 & basigin precursor & BSG & P3 & \\
\hline NP_001019820.1 & calnexin precursor & CANX & P3 & \\
\hline NP_006575.2 & T complex protein 1 subunit zeta 2 & CCT6B & P3 & \\
\hline NP_002942.2 & dolichyl diphosphooligosaccharide protein glycosyltransferase subunit 2 precursor & DDOST & P3 & \\
\hline NP_004721.1 & eukaryotic peptide chain release factor subunit 1 & ETF1 & P3 & \\
\hline NP_110416.1 & minor histocompatibility antigen $\mathrm{H} 13$ & HM13 & P3 & \\
\hline NP_006380.1 & hypoxia up regulated protein 1 precursor & HYOU1 & P3 & \\
\hline NP_005557.1 & L lactate dehydrogenase A chain & LDHA & P3 & \\
\hline NP_001244303.1 & lamin & LMNA & P3 & \\
\hline NP_000894.1 & NADPH dehydrogenase quinone 1 & NQO1 & P3 & \\
\hline NP_005023.2 & plastin 3 & PLS3 & P3 & \\
\hline NP_006397.1 & peroxiredoxin 4 precursor & PRDX4 & P3 & \\
\hline NP_001096137.1 & proteasome subunit alpha type 4 & PSMA4 & P3 & \\
\hline NP_653263.2 & proteasome subunit alpha type 7 like & PSMA7 & P3 & \\
\hline NP_055205.2 & staphylococcal nuclease domain containing protein 1 & SND1 & P3 & \\
\hline NP_110437.2 & thioredoxin domain containing protein 5 precursor & TXNDC5 & P3 & \\
\hline NP_066964.1 & $X$ ray repair cross complementing protein 5 & XRCC5 & P3 & \\
\hline
\end{tabular}

a crucial role in mediating stress in aging renal tubule epithelial cells.

Eukaryotic translation elongation factors are also closely related to senescence. It was reported that EEF1A1 and EEF1B2 could serve as senescence-associated biomarkers, which are downregulated during cellular senescence [14]. In this study, we confirmed that EEF1A1 and EEF2 were downregulated in senescent renal tubule cells (see Fig. 3). EEF1A1 is one of the alpha subunit forms of the elongation factor 1 complex that interacts with aminoacylated
tRNA and facilitates its delivery to the 'A' site of the ribosome during the elongation phase of protein synthesis. EEL1A1 is involved in moonlighting functions, including cytoskeletal remodeling, protein folding and degradation, cell signaling modulation, control of cell growth, apoptosis, and cell cycle. Therefore, our results also suggest that EEF1A1 may serve as a biomarker of renal tubule epithelial cell senescence.

More importantly, we explored molecular networks to define the role of biological functions in PTECs. In the 
Table 2 The upregulated DEPs in P6 tubule cells detected by LC-MS (Fold change > 1.5)

\begin{tabular}{|c|c|c|c|c|}
\hline Accession & Description & Protein & P6/P3 ratio & $\begin{array}{l}\text { Standard } \\
\text { deviation }\end{array}$ \\
\hline NP_001182032.1 & glutathione reductase mitochondrial isoform 3 precursor & GSR & 2.03 & 0.18 \\
\hline NP_001605.1 & actin cytoplasmic 2 & ACTG1 & 3.22 & 0.05 \\
\hline NP_001966.1 & gamma enolase & ENO2 & 3.74 & 0.45 \\
\hline NP_001093241.1 & POTE ankyrin domain family member $F$ & POTEF & 3.82 & 0.064 \\
\hline NP_001077007.1 & POTE ankyrin domain family member $E$ & POTEE & 3.90 & 0.065 \\
\hline NP_006363.4 & heterogeneous nuclear ribonucleoprotein Q & SYNCRIP & 4.10 & 0.27 \\
\hline NP_005991.1 & tubulin alpha $4 \mathrm{~A}$ chain & TUBA4A & 4.85 & 0.12 \\
\hline NP_001094.1 & alpha actinin 2 & ACTN2 & p6 & \\
\hline NP_001121089.1 & fructose bisphosphate aldolase $\mathrm{A}$ & ALDOA & p6 & \\
\hline NP_112092.1 & apolipoprotein L2 & APOL2 & p6 & \\
\hline NP_006076.4 & 325 bisphosphate nucleotidase 1 & BPNT1 & p6 & \\
\hline NP_775083.1 & calpastatin & CAST & p6 & \\
\hline NP_004850.1 & clathrin heavy chain 1 & CLTC & p6 & \\
\hline NP_000080.2 & collagen alpha 2 I chain precursor & COL1A2 & p6 & \\
\hline NP_444513.1 & dermcidin preproprotein & DCD & p6 & \\
\hline NP_004238.3 & 116 kDa U5 small nuclear ribonucleoprotein component & EFTUD2 & p6 & \\
\hline NP_001129490.1 & epoxide hydrolase 1 precursor & EPHX1 & p6 & \\
\hline NP_003079.1 & fascin & FSCN1 & p6 & \\
\hline NP_000138.2 & tissue alpha $L$ fucosidase precursor & FUCA1 & p6 & \\
\hline NP_000168.1 & gelsolin precursor & GSN & p6 & \\
\hline NP_003861.1 & ras GTPase activating like protein & IQGAP1 & p6 & \\
\hline NP_002435.1 & moesin & MSN & p6 & \\
\hline NP_038479.1 & myoferlin & MYOF & p6 & \\
\hline NP_060037.3 & $\mathrm{N}$ acetyl $\mathrm{D}$ glucosamine kinase & NAGK & p6 & \\
\hline NP_002769.1 & proactivator polypeptide preproprotein & PSAP & p6 & \\
\hline NP_002806.2 & 265 proteasome non ATPase regulatory subunit 11 & PSMD11 & p6 & \\
\hline NP_055113.2 & nicotinate nucleotide pyrophosphorylase carboxylating precursor & QPRT & p6 & \\
\hline NP_001003.1 & $40 \mathrm{~S}$ ribosomal protein $\mathrm{S} 8$ & RPS8 & p6 & \\
\hline NP_056456.1 & testin & TES & p6 & \\
\hline NP_001055.1 & transketolase & TKT & p6 & \\
\hline NP_001018005.1 & tropomyosin alpha 1 chain & TPM1 & p6 & \\
\hline NP_005992.1 & tubulin alpha 3 C D chain & TUBA3D & p6 & \\
\hline NP_997195.1 & tubulin alpha $3 E$ chain & TUBA3E & p6 & \\
\hline NP_006364.2 & synaptic vesicle membrane protein VAT 1 homolog & VAT1 & p6 & \\
\hline NP_003371.2 & vimentin & VIM & p6 & \\
\hline NP_001152994.1 & putative zinc finger protein 727 & ZNF727 & p6 & \\
\hline
\end{tabular}

network, translation elongation, stress, and glycolysis were associated with cytoskeleton regulation by PPI. We found that DEPs mediating cytoskeleton regulation were closely associated with regulating cell activities such as EMT and apoptosis in aging PTECs. For example, upregulation of DEPs VIM, IQGAP1, and moesin is closely related to EMT and renal fibrosis, [15, 16] and GSN, another DEP, is related to renal tubule epithelial cell apoptosis [17]. We deduced that translation elongation, stress, and glycolysis may regulate senescent cell activities such as apoptosis and EMT by influencing cytoskeleton regulation in PTECs [18-20]. EMT is a common change in cell phenotype of renal tubule epithelial cells, especially in those cells undergoing senescence. However, in this study, although P6 cells showed EMT-like characteristics (vimentin upregulation and E-cadherin 


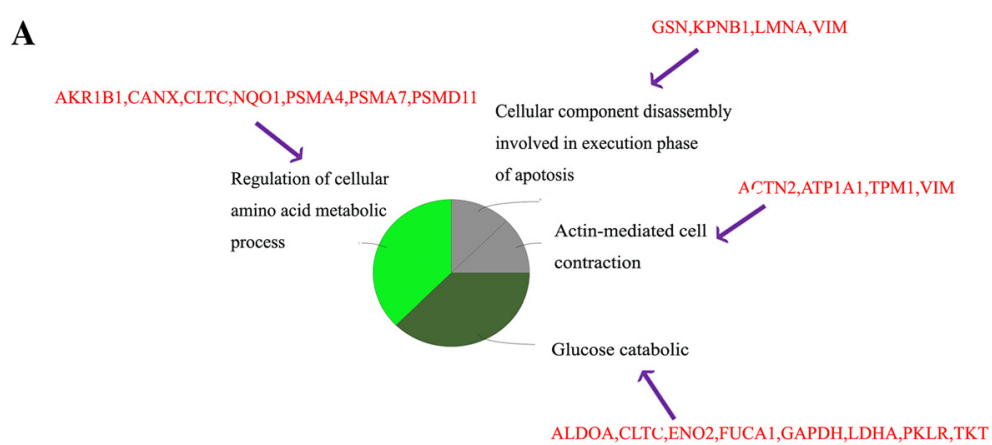

B

C

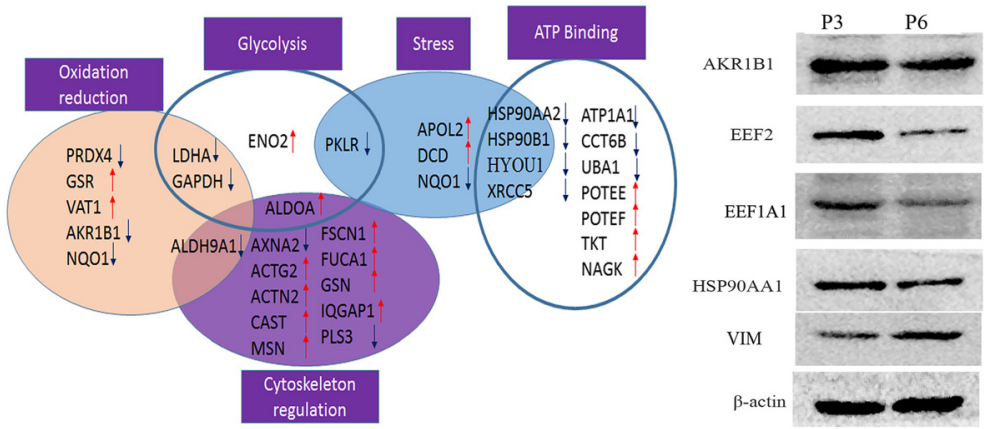

Fig. 2 Biological function analysis for proteomics profiles in P6 group. a CLUEGO analysis showed the DEPs in P6 group are involved in the regulation of cellular amino acid metabolic processes, apoptosis, actin-mediated cell contraction, and glucose catabolism. b BinGO analysis revealed additional biological functions, including glycolysis, response to stress, cytoskeleton regulation, oxidative reduction, adenosine triphosphate (ATP) binding, and oxidative stress. c the expression DEPs including AKR1B1, EEF2, EEF1A1 and HSP90 in the network was validated by western blot (Red up arrow meant DEPs upregulated in P6, and blue down arrow meant DEPs downregulated in P6)

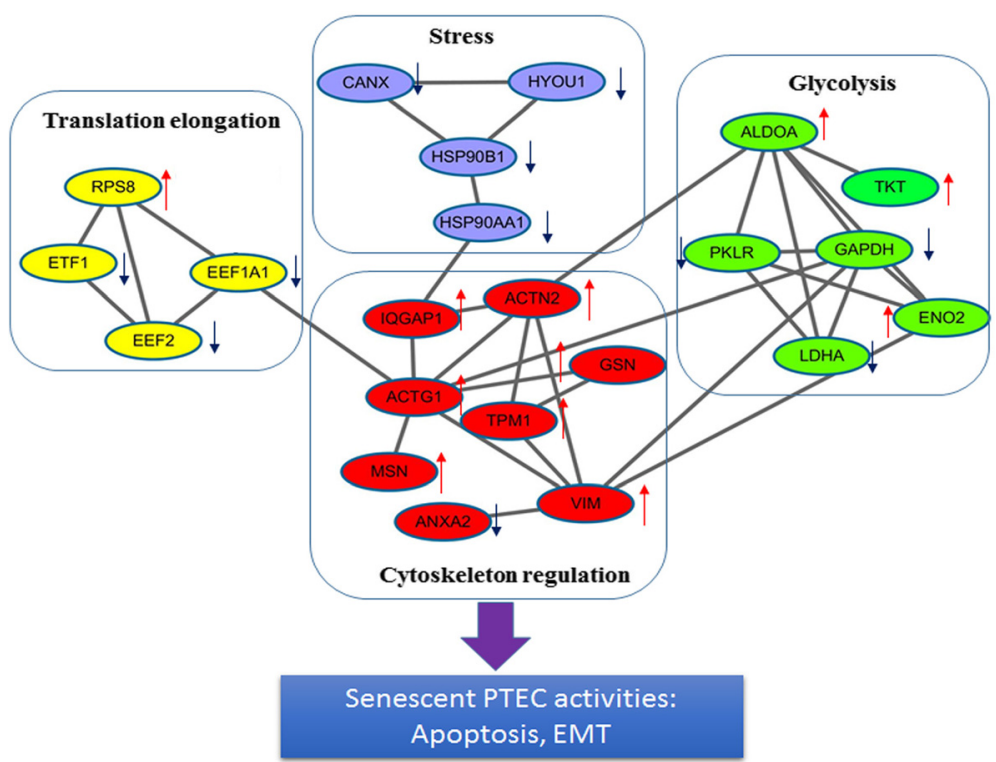

Fig. 3 Biological functions including translation elongation, stress and glycolysis could mediate the senescence-cell activities by acting on cytoskeleton regulation. The molecular network of DEPs was built by protein-protein interactions. In the network, RPS8, ETF1, EEF1A1, and EEF2 regulate eukaryotic translation elongation (Color: yellow), TKT, GAPDH, ALDOA, ENO2, LDHA, and PKLR are involved in glycolysis (Color: blue), DEPS, such as ACTN2, VIM, ANXA2, MSN, and GSM mediate cytoskeleton regulation (Color: red). CANX, HSP90B1, HSP90AA1 and HYOU1 are associated with oxidative stress (Color: green). Translation elongation, stress, glycolysis were all act on the cytoskeleton regulation, and in turn regulate cell activities in senescent PTEC (Red up arrow meant DEPs upregulated in P6, and blue down arrow meant DEPs downregulated in P6) 
downregulation), most cells maintained an epithelial cell morphology with CK18 expression. This result is supported by other reports, which showed that PTECs underwent EMT upon chemokine (ex. TGF- $\beta$ ) stimulation [21-23]. We deduce that most P6 cells cannot undergo EMT without a cytokine stimulus. This may help to explain why those exhibiting senescence in their kidneys may show greater renal fibrosis in the event of inflammation or nephrology.

\section{Conclusions}

We identified specific proteomic profiles involved in cell culture-induced senescence of renal tubule epithelial cells and built a senescence-associated biological function network involved in regulation of PTEC senescence activities. These results will aid in understanding the mechanisms involved in renal tubule epithelial cell senescence.

\section{Abbreviations \\ LC-MS: liquid Chromatograph Mass Spectrometer; EMT: epithelial-to- mesenchymal transition; PTEC: proximal tubule epithelial cells; DEP: differentially expressed protein; LDHA: L lactate dehydrogenase A chain; HYOU1: hypoxia up regulated protein 1 precursor; GAPDH: glyceraldehyde 3 phosphate dehydrogenase; PKLR: pyruvate kinase isozymes R L; LDHA: L lactate dehydrogenase A chain; HSP90B1: endoplasmin precursor; NQO1: NADPH dehydrogenase quinone 1; EEF1A1: elongation factor 1 alpha 1; VIM: vimentin; IQGAP1: ras GTPase activating like protein; GSN: gelsolin precursor.}

\section{Competing interests}

The authors declare that they have no competing interests.

\section{Authors' contributions}

XM Chen is the corresponding author and designed the experiments. Y Lu and JC Wang performed the proteomics experiment and completed the manuscript. DP Chen and Di Wu performed the cell biology experiments. Guangyan Cai analyzed the data. All authors have read and approved the final manuscript.

\section{Acknowledgments}

This work was supported by the National Natural Science Foundation of China (nos. 81330019 and 81270794), the National Basic Research Program of China (nos. 2013CB530800 and 2014CBA02005), and the Beijing Nova Program (nos. xx2014B096).

\section{Author details}

'Department of Nephrology, Chinese PLA General Hospital, Chinese PLA Institute of Nephrology, Beijing Key Laboratory of Kidney Disease, State Key Laboratory of Kidney Diseases, National Clinical Research Center for Kidney Diseases, General Hospital of PLA, Fuxing Road 28, Beijing 100853, P.R. China. ${ }^{2}$ Department of Nephrology, China-Japan Friendship Hospital, Beijing, China.

Received: 21 July 2015 Accepted: 18 March 2016

Published online: 01 April 2016

\section{References}

1. Gourtsoyiannis N, Prassopoulos P, Cavouras D, Pantelidis N. The thickness of the renal parenchyma decreases with age: a CT study of 360 patients. AJR Am J Roentgenol. 1990;155(3):541-4.

2. Liu Y. Cellular and molecular mechanisms of renal fibrosis. Nat Rev Nephrol. 2011;7(12):684-96.

3. Wiggins JE, Patel SR, Shedden KA, Goyal M, Wharram BL, Martini S, Kretzler M, Wiggins RC. NFkappaB promotes inflammation, coagulation, and fibrosis in the aging glomerulus. J Am Soc Nephrol. 2010;21(4):587-97.
4. Lu Y, Liu X, Shi S, Su H, Bai X, Cai G, Yang F, Xie Z, Zhu Y, Zhang Y, et al. Bioinformatics analysis of proteomic profiles during the process of anti-thy nephritis. Mol Cell Proteomics. 2012;11(4):M111 008755

5. Wang L, Hong Q, LV Y, Feng Z, Zhang X, Wu L, Cui S, Hou K, Su H, Huang Z et al. Autophagy can repair endoplasmic reticulum stress damage of the passive Heymann nephritis model as revealed by proteomics analysis. J Proteomics. 2012;75(13):3866-76.

6. Shen Z, Li P, Ni RJ, Ritchie M, Yang CP, Liu GF, Ma W, Liu GJ, Ma L, Li SJ et al. Label-free quantitative proteomics analysis of etiolated maize seedling leaves during greening. Mol Cell Proteomics. 2009;8(11):2443-60.

7. Xu D, Suenaga N, Edelmann MJ, Fridman R, Muschel RJ, Kessler BM. Novel MMP-9 substrates in cancer cells revealed by a label-free quantitative proteomics approach. Mol Cell Proteomics. 2008;7(11):2215-28.

8. Du S, Guan Z, Hao L, Song Y, Wang L, Gong L, Liu L, Qi X, Hou Z, Shao S. Fructose-bisphosphate aldolase $\mathrm{a}$ is a potential metastasis-associated marker of lung squamous cell carcinoma and promotes lung cell tumorigenesis and migration. PLoS One. 2014;9(1):e85804.

9. Tang H, Duan C, Bleher R, Goldberg E. Human lactate dehydrogenase A (LDHA) rescues mouse Ldhc-null sperm function. Biol Reprod. 2013;88(4):96.

10. Han SH, Malaga-Dieguez L, Chinga F, Kang HM, Tao J, Reidy K, Susztak K: Deletion of Lkb1 in Renal Tubular Epithelial Cells Leads to CKD by Altering Metabolism. J Am Soc Nephrol 2016:27(2):439-453.

11. Lindenmeyer MT, Rastaldi MP, Ikehata M, Neusser MA, Kretzler M, Cohen CD, Schlondorff D. Proteinuria and hyperglycemia induce endoplasmic reticulum stress. J Am Soc Nephrol. 2008;19(11):2225-36.

12. Islam A, Abraham $P$, Hapner CD, Andrews-Shigaki B, Deuster $P$, Chen $Y$. Heat exposure induces tissue stress in heat-intolerant, but not heat-tolerant, mice. Stress. 2013;16(2):244-53.

13. Song L, Robson T, Doig T, Brenn T, Mathers M, Brown ER, Doherty V, Bartlett JM, Anderson N, Melton DW. DNA repair and replication proteins as prognostic markers in melanoma. Histopathology. 2013;62(2):343-50.

14. Kim BC, Yoo HJ, Lee HC, Kang KA, Jung SH, Lee HJ, Lee M, Park S, Ji YH, Lee YS, et al. Evaluation of premature senescence and senescence biomarkers in carcinoma cells and xenograft mice exposed to single or fractionated irradiation. Oncol Rep. 2014;31(5):2229-35.

15. Wang XX, Wang K, Li XZ, Zhai LQ, Qu CX, Zhao Y, Liu ZR, Wang HZ, An QJ, Jing LW, et al. Targeted knockdown of IQGAP1 inhibits the progression of esophageal squamous cell carcinoma in vitro and in vivo. PLoS One. 2014; 9(5):e96501.

16. Chen YX, Zhang W, Wang WM, Yu XL, Wang YM, Zhang MJ, Chen N. Role of moesin in renal fibrosis. PLoS One. 2014:9(11):e112936.

17. Kothakota S, Azuma T, Reinhard C, Klippel A, Tang J, Chu K, McGarry TJ, Kirschner MW, Koths K, Kwiatkowski DJ, et al. Caspase-3-generated fragment of gelsolin: effector of morphological change in apoptosis. Science. 1997; 278(5336):294-8

18. Perez WB, Kinzy TG. Translation elongation factor $1 A$ mutants with altered actin bundling activity show reduced aminoacyl-tRNA binding and alter initiation via elF2alpha phosphorylation. J Biol Chem. 2014;289(30):20928-38.

19. Wojtera-Kwiczor J, Gross F, Leffers HM, Kang M, Schneider M, Scheibe R. Transfer of a Redox-Signal through the Cytosol by Redox-Dependent Microcompartmentation of Glycolytic Enzymes at Mitochondria and Actin Cytoskeleton. Front Plant Sci. 2012:3:284.

20. Richter-Landsberg C, Goldbaum O. Stress proteins in neural cells: functional roles in health and disease. Cell Mol Life Sci. 2003;60(2):337-49.

21. Chen $Y$, Luo $Q$, Xiong Z, Liang W, Chen L, Xiong Z. Telmisartan counteracts TGF-beta1 induced epithelial-to-mesenchymal transition via PPAR-gamma in human proximal tubule epithelial cells. Int J Clin Exp Pathol. 2012:5(6):522-9.

22. Hung TJ, Chen WM, Liu SF, Liao TN, Lee TC, Chuang LY, Guh JY, Hung CY, Hung YJ, Chen PY, et al. 20-Hydroxyecdysone attenuates TGF-beta1-induced renal cellular fibrosis in proximal tubule cells. J Diabetes Complications. 2012:26(6):463-9.

23. Wei MG, Sun W, He WM, Ni L, Yang YY. Ferulic acid attenuates TGF-beta1induced renal cellular fibrosis in NRK-52E cells by inhibiting Smad/ILK/Snail Pathway. Evid Based Complement Alternat Med. 2015;2015:619720. 